\title{
La cancérogenèse colique humaine et expérimentale
}

Le cancer colique est l'une des causes les plus importantes de mortalité dans le monde occidental. Des facteurs familiaux existent dans certains cas, le mieux étudié étant celui de la polypose colique familiale. S'y surajoute toujours l'effet carcinogène de substances dérivées de l'alimentation et de la prolifération microbienne. Les acides biliaires joueraient un rôle particulièrement important de «promoteurs » de la prolifération colique; leur insolubilisation partielle par du carbonate de calcium (c'est-à-dire de la craie) pourrait constituer une utile mesure préventive. Par ailleurs, la prophylaxie du cancer colique repose sur la détection et l'exérèse des polypes coliques qui précèdent le plus souvent le développement des cancers.

\section{Monique Martin \\ Directeur de recherche à l'Inserm}

\section{François Martin \\ Professeur à la faculté de méde- cine de Dijon}

\section{RÉFÉRENCES}

l. Faivre J, Milan C, Munsch P, Hillon P, Boutron MC, Klepping C Le cancer colorectal dans le département de la Côte-d'Or. Bull Cancer 1984; 71 : 50-6.

2. Laqueur GL. The induction of intestinal neoplasms in rats with the glucoside cycasin and its aglycone. Virchows Arch (Pathol Anat) $1965 ; 340: 151-63$.

3. Druckrey $\mathrm{H}$. Production of colonic carcinomas by 1,2-dialkylhydrazines and azoalkanes. In: Burdette WJ, Thomas CC, eds. Carcinoma of the Colon and Antecedent Epithelium. Huddersfield : Springfield, 1970 : 267-79.

\section{ADRESSE}

M. Martin, F. Martin : groupe de recherche sur les cancers digestifs, Inserm U.252, faculté de médecine, 21033 Dijon, France.

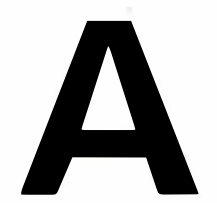

vec 15500 décès par an, les cancers du côlon et du rectum représentent en France la première cause de mortalité par cancer. Un français sur 25 sera atteint d'un cancer colorectal au cours de son existence. Il existe cependant des variations géographiques importantes dans l'incidence de ces cancers : dans le sexe masculin le taux standardisé pour 100000 habitants est de 2,1 pour le Sénégal et 37,2 pour la France [1]. Ces variations suggèrent l'importance des facteurs environnementaux, confirmés par les études épidémiologiques d'une part, les modèles expérimentaux de cancers colorectaux chimio-induits chez les rongeurs, d'autre part.

\section{Modèles expérimentaux de la cancérogenèse intestinale}

Les cancers intestinaux spontanés sont exceptionnels ches l'animal. Ilest en revanche possible de les induire chez le rat, la souris ou le hamster en utilisant des cancérogènes chimiques (figure 1, page 282). Les travaux de Laqueur[2] sur la cycasine, glucoside du méthylazoxyméthanol, et de Druckrey [3] sur les alkylhydrazines ont permis d'obtenir chez tous les animaux traités des cancers spécifiquement localisés à l'intestin, grêle et côlon. D'autres cancérogènes à spécificité intestinale élevée ont été découverts depuis, comme la méthylnitroso-urée et la $\mathrm{N}$-méthyl-N'-nitro-N-nitrosoguanidine utilisées par injection intrarectale. Ces produits sont des cancérogènes directs, ce qui explique leur efficacité locale et par là leur spécificité. D'autres, comme la 1,2-diméthylhydrazine ou l'azoxyméthane peuvent être administrés par voie générale. Ils sont transformés par le foie en dérivés activés, le cancérogène ultime n'apparaissant que dans la cellule intestinale dont l'équipement enzymatique conditionne la spécificité d'organe. Les tumeurs intestinales induites par ces cancérogènes sont des adénomes et des adénocarcinomes, histologique- 
ment très proches des tumeurs humaines correspondantes, mais sont généralement multiples (figures 2 et 3). Comme les tumeurs humaines homologues, les carcinomes intestinaux induits peuvent envahir les organes de voisinage et les ganglions mésentériques, ou donner des métastases à distance, en particulier aux poumons, mais exceptionnellement des métastases hépatiques. Les tumeurs intestinales chimio-induites permettent d'étudier les facteurs intervenant dans la cancérogenèse intestinale humaine: flore intestinale, acides biliaires, régime alimentaire, statut immunologique [4].

\section{Cancers colorectaux et lésions pré-cancéreuses}

Des modifications de l'épithélium intestinal peuvent précéder, chez l'homme, l'apparition d'un cancer colorectal. Les polypes adénomateux résultent d'une prolifération néoplasique, mais bénigne, des cellules épithéliales de l'intestin. Ces tumeurs sessiles ou pédiculées, uniques ou

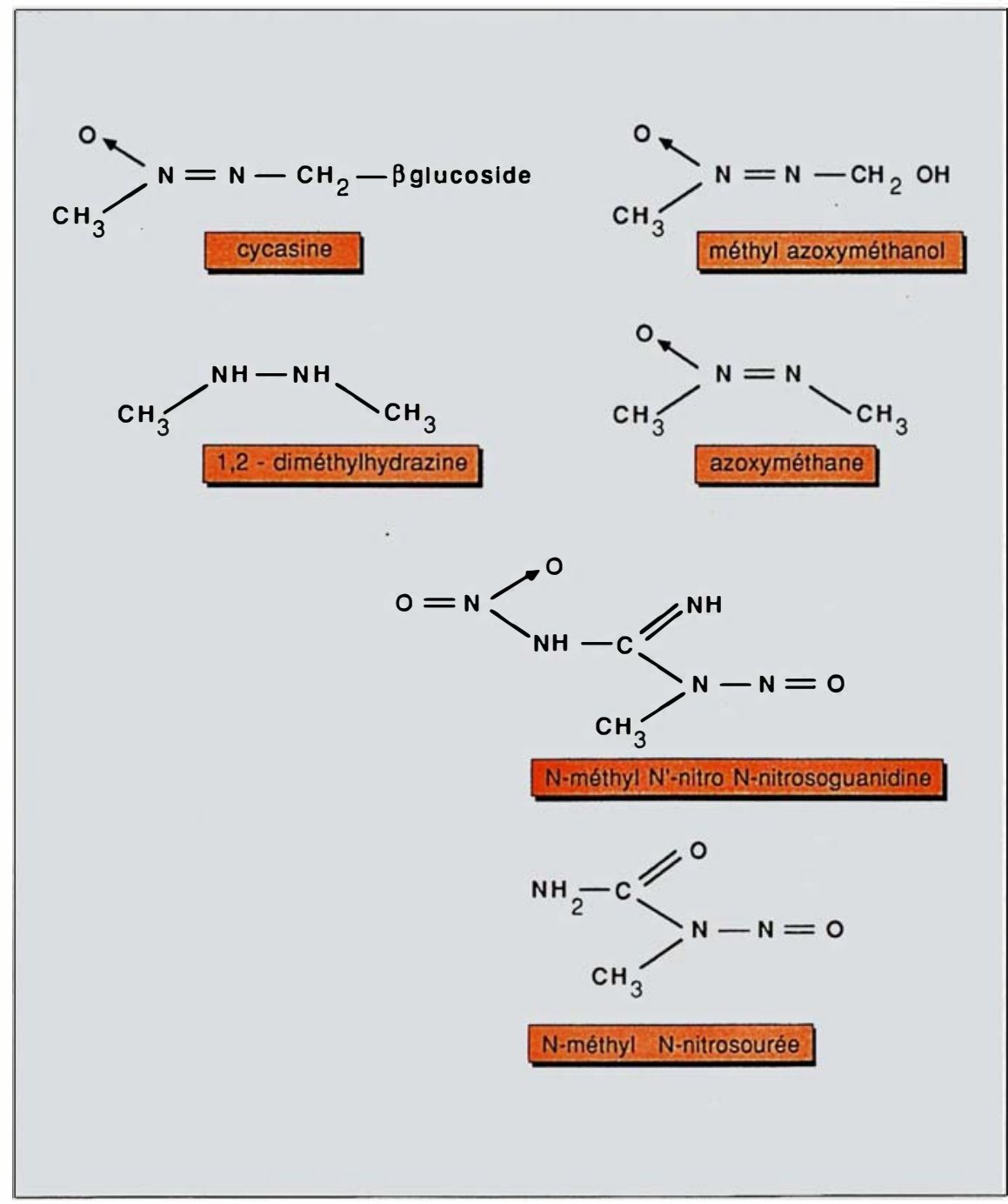

Figure 1. Structure de quelques molécules capables d'induire des cancers de l'intestin chez le rat ou la souris. multiples, dont la dimension varie de quelques millimètres à plusieurs centimètres sont des adénomes tubuleux, les plus fréquents, des adénomes villeux, ou des adénomes tubulo-villeux. Ils peuvent être bien différenciés ou dysplasiques. La prévalence des polypes est d'environ $10 \%$ dans la population française entre 45 et 70 ans. Les polypes adénomateux peuvent se transformer en cancers intestinaux. La preuve la plus directe est la constatation fréquente de résidus adénomateux au sein des petits cancers de l'intestin ou la découverte de foyers tumoraux dans les polypes adénomateux (figure 4), découverte d'autant plus fréquente que les polypes sont plus volumineux, qu'ils comportent un important contingent villeux, ou qu'ils sont plus dysplasiques. La majorité des cancers intestinaux provient de la transformation d'un polype [5]. Mais il existe aussi des cancers de novo surtout au niveau du côlon droit et du rectum. Le temps séparant l'apparition d'un polype et sa transformation en cancer peut être estimé à un minimum de cinq ans, parfois plus de 20 ans. Des polypes adénomateux, très proches des polvpes adénomateux humains, peuvent être induits chez les rongeurs par les mêmes cancérogènes chimiques que les carcinomes colorectaux. Ces polypes adénomateux sont fréquemment observés chez la souris, plus rarement chez le rat. La transformation des polypes adénomateux en cancers ne paraît pas être un événement fréquent : la plupart des cancers colorectaux chimio-induits chez l'animal apparaissent de novo.

La rectocolite hémorragique peut se compliquer d'un cancer colorectal, surtout après dix ans d'évolution. Ces cancers sont précédés de lésions dysplasiques planes. Ils ne représentent qu'une très faible fraction des cancers colorectaux observés dans l'ensemble d'une population.

Les travaux de Lipkin ont permis de montrer qu'une autre anomalie pouvait être fréquemment associée aux cancers colorectaux ou précéder leur apparition : une prolifération anormale de l'épithélium colorectal sans anomalie morphologique détectable par les techniques histologiques habituelles [6]. Le renouvellement de l'épithélium colique normal est 
Figure 2. Cancers coliques induits chez le rat par la 1,2-diméthylhydrazine. Deux cancers sont visibles (indiqués par les deux flèches noires) sur ce segment de côlon.

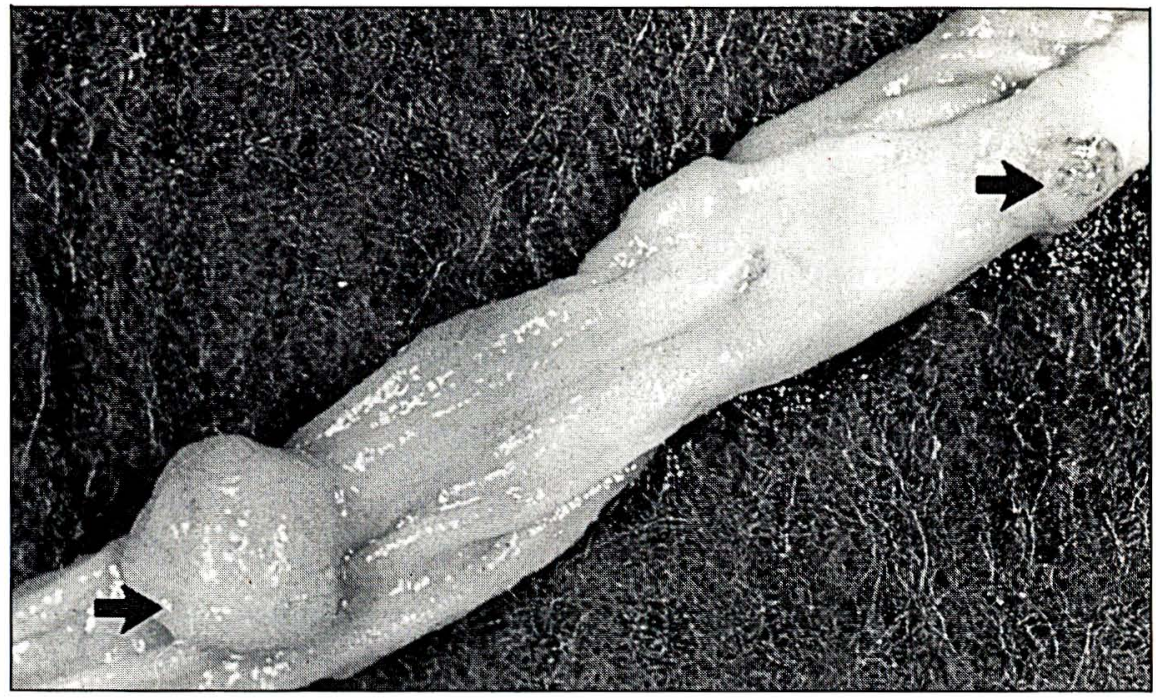

Figure 3. Cancers coliques induits chez le rat par la 1,2-diméthylhydrazine. Coupe histologique. A gauche, intestin normal. Les cinq flèches noires en bas délimitent la muscularis mucosae, interrompue par la tumeur qui occupe la partie droite de la figure. En haut, à gauche $: L=$ lumière de l'intestin.

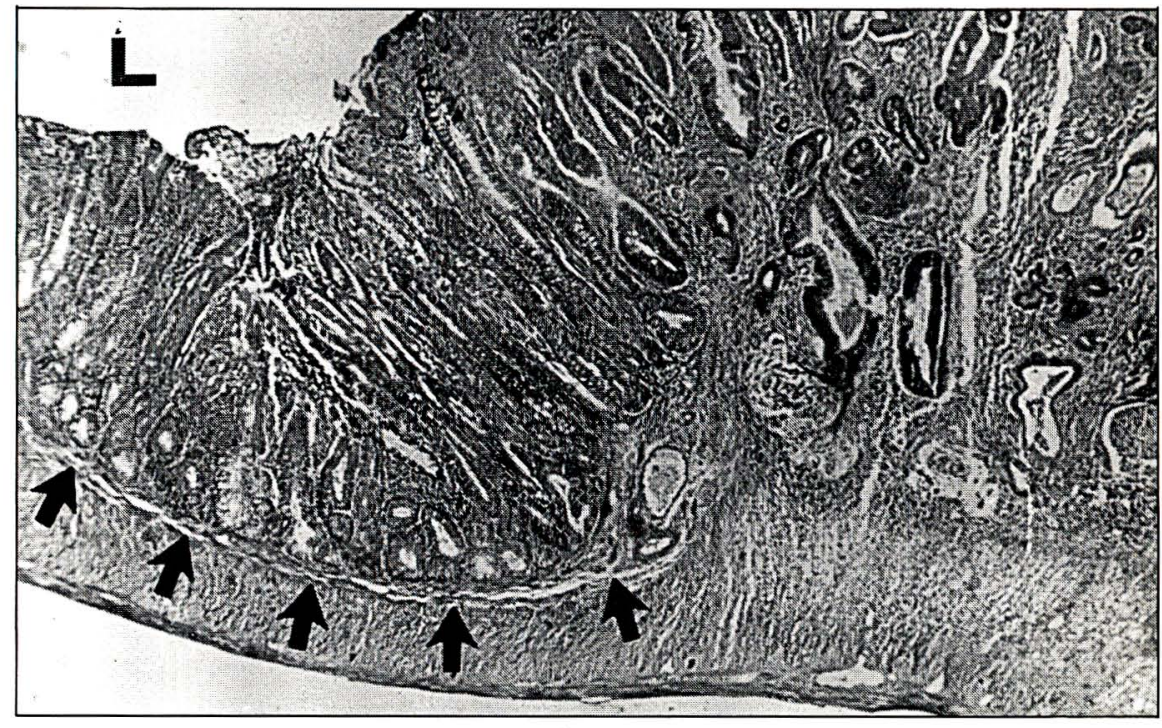

Figure 4. Polype adénomateux humain de type tubulaire contenant une zone transformée $(C)$ par un adénocarcinome.

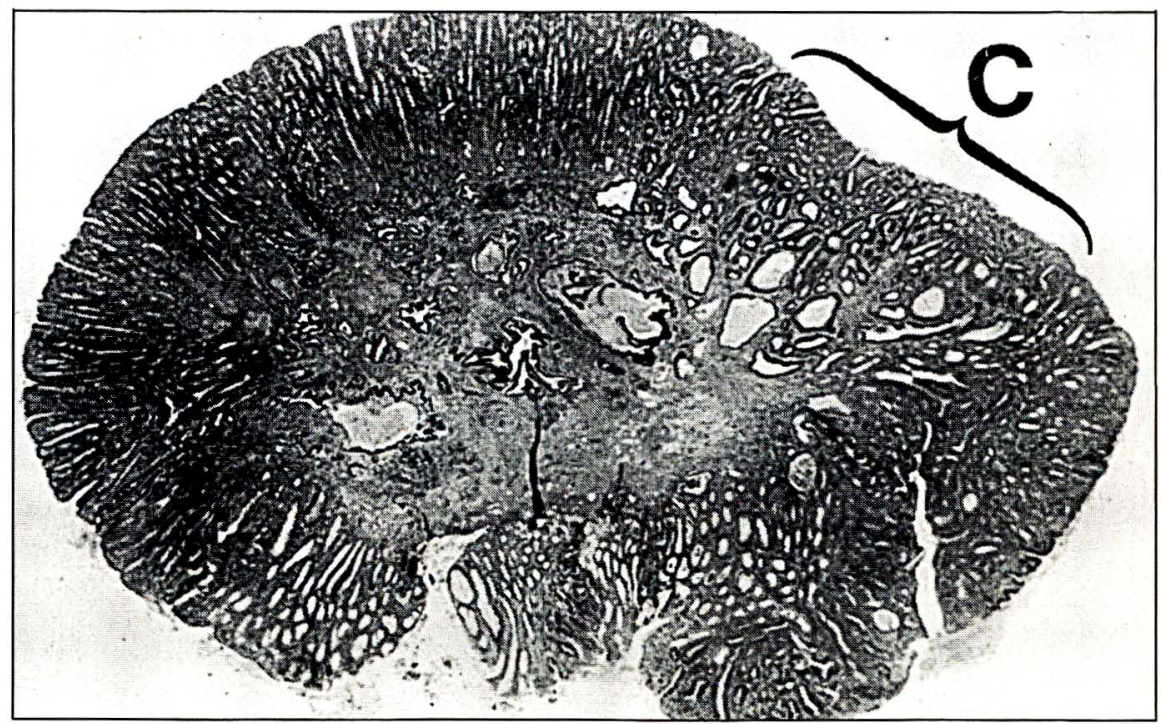




\section{RÉFÉRENCES}

4. Martin MS. Cancérogenèse colique expérimentale. Gastroenterol Clin Biol 1982; 6 . 382-91.

5. Morson BC. The pathogenesis of colorectal cancer. Monograph. vol. 10 of Major Problems in Pathology. Philadelphia : Saunders, 1978.

6. Lipkin M, Blattner W, Fraumeni J, Lynch $H$, Deschner EE, Winawer S. Tritiated thymidine labeling distributions in the identification of hereditary predisposition to colon cancer. Cancer Res 1983; 43 : 1899-904.

7. Maire P, Morichau-Beauchant M, Drucker J, Barboteau MA, Barbier J, Matuchansky C. Prévalence familiale du cancer du côlon et du rectum: résultats d'une enquête «castémoins " de 3 ans. Gastroenterol Clin Biol $1984 ; 8: 22-7$.

8. Bodmer WF, Bailey CJ, Bodmer J, et al. Localization of the gene for familial adenomatous polyposis on chromosome 5. Nature 1987; $327: 614-16$.

9. Solomon E, Voss R, Hall V, et al. Chromosome 5 allele loss in human colorectal carcinomas. Nature 1987 ; 328 ; 616-9.

10. Bos JL, Fearon ER, Hamilton SR, et al. Prevalence of ras gene mutations in human colorectal cancers. Nature 1987 ; 327 : 293-7.

11. Forrester K, Almoguera C, Han K, Grizzle $W E$, Perucho $M$. Detection of high incidence of K-ras oncogenes during human colon tumorigenesis. Nature 1987 ; 327 : 298-303.

12. Bruce WR. Recent hypotheses for the origin of colon cancer. Cancer Res 1987; 47 : 4237-42.

13. Reddy BS, Watanabe $\mathrm{K}$, Weisburger JH, Wynder EL. Promoting effec of bile acids in colon carcinogenesis in germ free and conventional F 344 rats. Cancer Res $1977 ; 37$ : 4156-9.

14. Martin MS, Justrabo E, Jeannin JF, Leclerc A, Martin F. Effect of dietary chenodeoxycholic acid on intestinal carcinogenesis induced by 1,2 dimethylhydrazine in mice and hamsters. Br J Cancer 1981 ; 43 : 884-6.

15. Drasar BS, Hill MJ. Human intestinal flora. London: Academic Press, 1974.

16. Hill MJ. The role of colon anaerobes in the metabolism of bile acids and steroids, and the metabolism of bile acids and steroids, and 2387-400.

17. Reddy BS, Watanabe K, Weisburger J. Effect of high fat diet on colon carcinogenesis in F 344 rats treated with 1,2 dimethylhydrazine, methylazoxymethanol acetate or methylnitrosourea. Cancer Res 1977 ; 37 : 4156-9.

18. Wargovich MJ, Eng VWS, Newmark HL, Bruce WB. Calcium ameliorates the toxic effect of deoxycholic acid on colonic epithelium. Carcinogenesis 1983 ; 9 : 1205-7.

19. Martin MS, Hammann A, Martin F. Gut associated lymphoid tissue and 1.2 dimethylhydrazine intestinal tumors in the rat: an histological and immunoenzymatic study. Int J Cancer 1986 ; 38 : 75-80.

20. Lipkin M, Newmark H. Effect of added dietary calcium on colonic epithelial cell proliferation in subjects at high risk for familial colonic cancer. $N$ Engl J Med 1985; 313 : assuré par une prolifération cellulaire localisée aux deux tiers profonds des cryptes coliques. Une extension vers la surface de la zone proliférative a été trouvée chez les sujets normaux présentant un risque génétique de cancer colorectal, ainsi que chez les animaux traités par des cancérogènes intestinaux ou exposés à des concentrations élevées d'acides biliaires.

\section{Existe-t-il un gène du cancer colorectal?}

Les enquêtes familiales montrent que le risque de cancer colorectal est plus important chez les proches parents d'un sujet atteint par cette maladie. Dans un travail réalisé à Poitiers en 1984, les antécédents de cancer colorectal chez les parents au premier degré sont 5,3 fois plus fréquents chez les patients atteints d'un cancer colorectal que dans un groupe apparié de sujets témoins [7].

Dans certaines familles, le risque est beaucoup plus élevé. C'est le cas des familles, d'abord décrites chez les mormons de l'Utah dans lesquelles plusieurs membres d'une même génération ou de générations successives sont atteints de cancers du côlon proximal, cancers survenant souvent très tôt, entre 30 et 50 ans. Mais c'est dans la polypose familiale que l'hérédité des cancers colorectaux s'exprime sous sa forme majeure. Dans ces familles un gène autosomique dominant détermine l'apparition chez la moitié des descendants d'une polypose diffuse, recouvrant la muqueuse colorectale d'un tapis de polypes adénomateux. Ces adénomes peuvent apparaître dès la fin de l'adolescence, restent souvent latents jusqu'à l'âge de 30 ou 40 ans lorsque plusieurs d'entre eux se transforment inéluctablement en cancers.

C'est en appliquant les méthodes de la génétique moléculaire à l'étude de la polypose familiale que l'équipe du Pr. Bodmer à Londres a récemment pu mettre en évidence une anomalie génétique de l'un des deux chromosomes 5 [8]. L'analyse de familles atteintes de polypose familiale colique a révélé une relation entre l'expression de la polypose et l'absence d'un fragment d'ADN reconnu par la sonde Cllpll et loca- lisé à une région précise (5q2l-q22) du chromosome 5 . Ce fragment peut exister sous deux formes moléculaires différentes qui peuvent être présentes dans l'ADN d'un même sujet si les deux chromosomes 5 contiennent chacun une forme différente ${ }^{*}$. L'ADN des malades atteints de polypose familiale ne contient jamais simultanément les deux formes moléculaires. Cette constation implique la délétion de la région contenant le fragment reconnu par la sonde Cllpll sur l'un des deux chromosomes 5 de toutes les cellules de l'organisme. Cette région contiendrait un gène régulateur, contrôlant la prolifération de la muqueuse colique. Cette prolifération n'est normale que si les deux gènes régulateurs (un par chromosome 5) sont présents et fonctionnels. Dans la polypose familiale la délétion de l'un des deux gènes provoquerait la prolifération diffuse de la muqueuse colorectale. La transmission de cette anomalie dans la descendance est compatible avec la transmission autosomique et dominante de la polypose familiale.

La même équipe a montré une délétion de la même région du chromosome 5 chez 20 à $40 \%$ des malades atteints d'un cancer colorectal «banal». L'anomalie n'est présente que dans les cellules tumorales. Le cancer résulterait de deux événements successifs: une mutation ponctuelle frappant une cellule de l'épithélium colique inactiverait le gène de contrôle de l'un des deux chromosomes 5 , provoquant l'apparition d'un polype adénomateux. $\mathrm{La}$ transformation du polype en cancer serait due à la perte du fragment chromosomique porteur du gène de contrôle resté fonctionnel sur le chromosome 5 non muté de l'une des cellules de l'adénome [9].

\section{Cancer colorectal et oncogènes}

L'utilisation de sondes spécifiques montre la fréquence d'une mutation ponctuelle des proto-oncogènes de la famille ras dans les cancers colorectaux humains $[10,11]$, le plus sou-

\footnotetext{
* Il s'agit d'un polymorphisme de restriction.
} Voir RFLP (m/s suppl. au $n^{\circ} 7$, vol. 3, p. 4). 
vent une substitution d'un acide aminé à la glycine en position 12 de la protéine 21 codée par l'oncogène $K i$-ras2. Cette mutation a aussi été trouvée dans des résidus adénomateux inclus dans les cancers colorectaux suggérant l'activation de l'oncugène dès le stade de la tumeur bénigne. L'expression de l'oncogène $m \nu c$ et des oncogènes de la famille ras est souvent exagérée dans les cancers colorectaux et dans les polypes adénomateux. Cette expression exagérée ne parait être due ni à une amplification ni à une translocation des gènes correspondants.

\section{Mutagènes et cancérogènes dans la lumière colique}

Les cellules de l'épithélium colique à partir desquelles naissent les cancers colorectaux sont en contact intime avec le contenu intestinal. Il était donc logique de rechercher dans les matières fécales des substances chimiques cancérogènes ou mutagènes. Trois groupes de substances ont été particulièrement étudiés : les produits de la pyrolyse des aliments, les fécapentaènes et les stéroïdes cytotoxiques [12]. Les aliments exposés à des températures élevées (fritures, grillades) génèrent des produits de pyrolyse capables de provoquer des mutations dans des systèmes bactériens (test de Ames) ou dans des cellules de mammifères. Plusieurs de ces produits comme le 2-amino-6 méthyldipyrido-imidazole ou la 2amino-3-méthylimidazoquinoline provoquent des aberrations nucléaires chez la souris, des tumeurs intestinales bénignes ou malignes chez le rat. En utilisant le test de Ames, Bruce et son équipe ont mis en évidence dans les matières fécales humaines, une activité mutagène due à des éthers de glycérol et d'alcools polyinsaturés comportant cing doubles liaisons, d'où leur nom de fécapentaènes. Les fécapentaènes, produits par certaines souches de Bacteroides présents dans la flore intestinale, sont très instables, ce qui rend difficile la recherche de leur pouvoir carcinogène chez l'animal. Un troisième groupe de cancérogènes potentiels a été trouvé dans les matières fécales humaines en purifiant les substances capables d'in$\mathrm{m} / \mathrm{s} n^{\circ} 5$ vol. 4 , mai 88 duire des aberrations nucléaires après instillation locale dans la muqueuse colique de souris. Le groupe de Bruce a aussi isolé et caractérisé deux stéroïdes cytotoxiques, le 5-cholestan-3-one et le cholest-4-en-3-one mais leur carcinogénicité chez l'animal n'a pas encore été étudiée. Bien que le rôle des trois groupes de substances dans la cancérogenèse colique humaine n'ait pas été directement prouvé, les cellules de l'épithélium colique sont donc en contact direct avec plusieurs cancérogènes ou mutagènes potentiels.

\section{De puissants promoteurs :} les acides biliaires

Dans de nombreux modèles expérimentaux, l'apparition d'une tumeur résulte de l'action d'un initiateur, généralement un mutagène, et d'un promoteur non mutagène. La promotion des cancers intestinaux paraît assurée par les acides biliaires du contenu intestinal. La foie synthétise deux acides biliaires primaires, les acides cholique et chéno-désoxycholique dont près de $90 \%$ sont réabsorbés dans le grêle puis à nouveau sécrétés dans la bile. Les $10 \%$ restants arrivent dans le côlon et sont déshydroxylés par la flore intestinale en acides biliaires secondaires, acides désoxycholique et lithocholique. Les acides biliaires secondaires augmentent la prolifération des cellules intestinales, ce qui explique probablement leur effet promoteur sur les cancers coliques. Les cancers intestinaux sont significativement plus nombreux chez les rats ou les souris qui reçoivent en lavement ou par voie orale de l'acide désoxycholique ou de l'acide lithocholique [13]. Chez l'homme, il y a une relation significative entre la concentration en acides biliaires secondaires dans les matières fécales et le risque d'adénomes et de cancers colorectaux. L'acide lithocholique, produit de dégradation par les bactéries intestinales de l'acide chénodésoxycholique, semble particulièrement nocif. L'acide chénodésoxycholique a été utilisé dans le traitement de la lithiase vésiculaire chez l'homme et son administration à des doses équivalentes chez la souris augmente significativement l'incidence des tumeurs intestinales provoquées par la 1,2-diméthylhydrazine [14].

\section{Rôle de la flore bactérienne et de l'alimentation}

Les cancers colorectaux naissent et se développent au contact direct du contenu intestinal. Celui-ci est caractérisé, dans le côlon et le rectum, par une abondante flore bactérienne hétérogène constituée surtout de bactéries anaérobies strictes [15]. La flore bactérienne joue un rôle important dans la genèse ou dans le métabolisme des agents cancérogènes et mutagènes et dans la transformation des acides biliaires primaires en acides biliaires secondaires, promoteurs actifs de la cancérogenèse intestinale [16].

Le rôle des régimes alimentaires dans la cancérogenèse colique humaine est suggéré par les études épidémiologiques qui opposent les populations à risque élevé de cancer ou d'adénome colorectal, dont l'alimentation est riche en graisses, pauvre en fibres végétales, et les populations à faible risque dont les caractéristiques alimentaires sont inverses. Dans les populations migrantes, le risque de cancer colorectal augmente parallèlement à l'adoption d'une nourriture de style «occidental». Les modèles expérimentaux confirment ces données : les cancers coliques induits par les cancérogènes chimiques sont significativement plus fréquents chez les rats qui reçoivent un régime riche en graisses: le caractère saturé ou insaturé des graisses, leur origine animale ou végétale n'ont qu'une importance marginale [17]. Les cancers chimio-induits sont moins fréquents chez les animaux dont le régime est additionné de son ou de cellulose, mais plus fréquents si un extrait d'algues marines, la carragénine, est utilisé comme «fibre » végétale. Les mécanismes par lesquels le régime alimentaire modifie la carcinogenèse colique sont complexes. Le régime modifie la composition de la flore bactérienne intestinale. Mais l'action prédominante s'exerce sur les acides biliaires : il existe une corrélation entre les lipides totaux alimentaires et la concentration des acides biliaires secondaire dans les matières fécales. Les fibres alimentaires absorbent les acides biliaires et les cancérogènes intestinaux et les diluent en augmentant le volume des 
matières fécales.

L'attention a été récemment attirée sur le rôle protecteur du calcium alimentaire [18]. L'adjonction de carbonate de calcium au régime diminue, chez l'homme, la prolifération des cellules coliques, réduit chez les rongeurs les effets prolifératifs des régimes riches en graisse ou des acides biliaires et l'incidence des cancers intestinaux chimio-induits. Le calcium agirait en insolubilisant les acides biliaires supprimant ainsi leur effet promoteur sur la cancérogenèse colique.

\section{Les facteurs immunologiques}

La muqueuse colique est riche en cellules et en formations lymphatiques qui peuvent jouer un rôle dans la cancérogenèse intestinale. La plupart des cancers chimio-induits chez le rat et la souris apparaissent au sein des formations lymphoïdes qui parsément le côlon et le rectum [19]. Le côlon humain est également riche en formations lymphoïdes au contact desquelles on peut observer des tubes glandulaires dysplasiques. Les mécanismes qui interviennent dans cette association sont encore inconnus.

\section{Implications pour la prévention des cancers}

La cancérogenèse colorectale résulte donc de facteurs multiples, certains endogènes, d'autres exogènes. Le polype adénomateux est l'étape habituelle entre la muqueuse normale et le cancer. Un premier mode de prévention consiste donc à réséquer les polypes adénomateux avant leur transformation en cancer. Mais les polypes sont généralement latents. Les plus volumineux peuvent cependant être à l'origine d'hémorragies microscopiques détectées par la recherche de sang dans les selles. L'efficacité de cette méthode dans la prévention des cancers colorectaux n'a pas encore été prouvée à l'échelle d'une population. Un autre mode de prévention consiste à diminuer les facteurs exogènes de la cancérogenèse. Une modification drastique des habitudes alimentaires paraît difficilement réalisable. Il serait plus faci- ration alimentaire quotidienne deux ou trois grammes de carbonate de calcium, produit peu onéreux (c'est le composant essentiel de la craie), capable de normaliser la prolifération de la muqueuse colique précancéreuse en bloquant l'effet irritatif des acides biliaires [20]

\section{${ }^{*}$ GLOSSAIRE ${ }^{*}$}

Adénome: tumeur bénigne pédiculée ou sessile de l'épithélium glandulaire présentant des atypies de degré variable.

Adénome tubulaire: tumeur bénigne composée de façon prédominante de tubules ramifiés enchassés dans la lamina propria ou entourés par elle.

Adénome villeux: tumeur bénigne composée de prolongements digitiformes pointus ou arrondis. Dans chacun d'eux une. expansion de la lamina propria est recouverte par l'épithélium qui descend jusqu'à la muscularis mucosae.

Adénome tubulo-villeux ou mixte: adénome pouvant présenter à la fois des structures tubulaires et villeuses ou une structure intermédiaire.

Lamina propria : tissu conjonctif de soutien remplissant les espaces entre les glandes intestinales ou les cryptes coliques.

Muscularis mucosae: fine couche de muscle lisse séparant la muqueuse de la sous-muqueuse. Rectocolite ulcéro-hémorragique: maladie inflammatoire d'origine inconnue du côlon, prédominant sur le rectum, respectant l'intestin grêle, traduite par un oedème et une vasodilatation intense de la muqueuse, provoquant des hémorragies et une hypersécrétion de mucus.

Test de Ames: test permettant d'évaluer le pouvoir mulagène d'un produit par son effet sur certaines souches de bactéries (Salmonelles) qui, mutées, acquièrent la capacité de se multiplier dans des milieux de culture définis.

\section{Summary}

Colorectal cancer is one of the most frequent cause of death in the Western countries. It results from multiple factors, either endogenous or environmental : deletion of genes controlling the proliferation of colorectal mucosa, activation of protooncogenes by mutagenic or genotoxic substances present in the diet or the intestinal content, secondary bile acids acting as tumor promoters. Epidemiologic study in the humans, and chemically-induced colorectal tumors in the rodents give information on the role of the intestinal microflora and dietary factors in the genesis of colorectal cancer. Since human colorectal carcinoma is often preceeded by a long-lasting benign lesion, the adenomatous polyp, colorectal cancer prevention could be obtained by a systematic detection and removal of the intestinal polyps. An alternative way could be the primary reduction of colorectal carcinogenesis by a modification of its factors, for instance through a supplementation of diet by calcium carbonate which insolubilizes the secondary bile acids.

\section{TIRÉS A PART}

M. Martin : groupe de recherche sur les cancers digestifs, Inserm U.252, faculté de médecine, 21033 Dijon, France.

$$
\mathrm{m} / \mathrm{s} n^{\circ} 5 \text { vol. 4, mai } 88
$$

